\title{
Role of rice paddy fields in our finite planet earth
}

\author{
Masaaki Hosomi
}

Published online: 16 May 2009

(C) Springer-Verlag 2009

Our ability to control our climatic and financial well-being is being severely challenged, as the IPCC Fourth Evaluation Report of 2007 and the recent global financial crises will testify. At the Summit at Toyo Lake in Hokkaido, Japan in 2008, the delegates gathered to discuss what could be done to tame the global warming phenomenon and the dramatic rise in food and oil prices resulting from globalization. The Japanese Government ratified in June 2007 a strategic plan to make Japan an environmental country for the twenty-first century, as a result of which the Ministry of Environment is committed to the development of what could be called a "low carbon society" or "natural symbiotic society" or "recycle society".

The university community has a central role in educating the future leaders of society, especially in environmental science and research so as to enable them to tackle the difficult and complex problems facing us now and in the future. On this point, in this article, I will offer my thoughts on how to deliver practical solutions for many of these problems. In several courses on the environmental science and engineering that I have organized over the years, I have always emphasized lessons learned from natural processes. The most useful source of my inspiration came from the book "Finite Ecology-Stable and Symbiotic system - " by Professor Yasushi Kurihara, which has been re-published as Doujidai-Library194 by Iwanami Publisher. Indeed, more than 30 years ago, when I was a graduate student, Professor Sueshi, my teacher of the ecology class elaborated on the idea of finite ecology as

\section{Hosomi ( $\square)$}

Department of Chemical Engineering, Tokyo University of Agriculture and Technology, 2-24-16 Nakamichi, Koganei, Tokyo 184-8588, Japan

e-mail: hosomi@cc.tuat.ac.jp applied to human growth and sustainability. The theory of environmental carrying capacity was central to finite ecology. Since then finite ecology has become my favorite obsession in my teaching and research.

Professor Kurihara has experimentally elucidated the intriguing phenomenon that many organisms can survive in a flask where no substrate is supplied. He has named the system of organisms that coexist on subsistent level in this way the co-existence and co-poverty system. Furthermore, Professor Kurihara has proposed other systems, for example, the co-existence and co-prosperity system such as exists in cow rumens, or a system where tension exists always among the constituent members, such as would occur in a space ship.

Coming back to the co-poverty system, bacteria first proliferate in a flask under sun light, followed by growth of protozoa. However, as the available substrate is consumed concurrent with a decrease in protozoa, the water surface becomes transparent and changes to green because of chlorella growth by sunlight. After a while, chlorella decrease due to shortage of nutrients, resulting in growth of rotifers in the bulk and of filamentous blue-green algae. As the microorganisms repeat growing and decaying, this ecosystem leads to a steady-state where they stably coexist for a long time. In other words, a sustainable microcosm emerges in the flask. Although a single microorganism decays after substrates are depleted, why can these microorganisms survive in the flask for a long period? Development of such a sustainable microcosm is not created by us; the microorganisms spontaneously design the system in the flask based on light energy and nutrient concentrations in the flask. We can, therefore, learn the basics of material recycling and the energy flow in the natural systems. In addition to the recycle system intertwined with the mechanistically balanced interaction 
among microorganisms, we realize that each constituent microbial species in the system needs to reduce its energy to ensure the stability and co-existence among the microorganisms. The name "co-poverty system" is derived from this phenomenon.

Taking a look at a field of rumen, a cow continuously triturates grasses and provides them to the rumen where highly active anaerobic microbes and protozoa are inhabited under controlled environmental conditions, viz. temperature and $\mathrm{pH}$. In light of such perpetual substrate supply, a rumen is an example of the co-existence and co-prosperity system. Human systems are analogous to this type of system. We, indeed, have been developing the co-existence and co-prosperity system based on mass-production and consumption using numerous energy resources.

Needless to say, it is difficult to achieve such a co-existence and co-prosperity system with limited oil resources. Hence we have been pointing out the significance to consider our planet to represent symbiosis: a term for co-existence and co-prosperity with some limitation. From the perspectives of mass balance, energy and subsistence of individual organisms, Professor Kurihara has proposed the hypothesis that the limited resources and environment for human being is similar to a space base. The space base is a system where all organisms, including human beings, have relations of sustainable symbiosis. Hence, substances and energies smoothly flow in a closed system with the mass balances completely maintained, resulting in selection of suitable constituents for such an environment and steady maintenance of their activities when not buffeted by external disturbances. As a result, spontaneity of each constituent is restricted and its behavior is systematized and simplified. Such simplification strengthens the interactive dependency of each constituent, compromising the balance of each constituent. In other words, the system lacks stability against outer perturbations.

Questions can be asked about how can a system be sustainable, and which direction it has to head for. An artificial system, e.g., a city, needs to attain spontaneity not just from material and energy but, as Professor Kurihara has argued, also from cultural traits such as societal value systems and behavior of cultures.

Despite being raised in a farm family, I have never done agriculture work. However, I was, by chance, forced to do the work 4 years ago when my father passed away. Apart from the productivity of agriculture practice, a sense of soils and vital energy of plants really touched me at that time. Nevertheless, this was a disappointing experience because of a series of failures punctuated by occurrences of typhoons. I learnt that one-crop succession is not suitable for black beans when the second year yield was dismal. Rice plants, on the other hand, do not suffer from this deficiency although a certain amount of herbicides and fertilizers is, of course, necessary. This simply gave me a clue that rice paddy incorporated with rice plants could be a key to a sustainable crop system. The memory of this experience reminded me again that there is so much to learn from the workings of natural systems, as is evidenced by my present preoccupation with eutrophication control measures.

Up to now, my research group has been focusing on purification capability of aquatic plants, e.g., reed. It has been demonstrated that reed rhizosphere has the spot for denitrification, where denitrifying bacteria can grow, and that the produced nitrogen gas is discharged from the rhizome to the atmosphere via the terrestrial stem, which indicates that reeds are potentially capable of sustainable nitrogen removal. Indeed, reconstructive projects for waterside and water purification (vegetative purification) by means of plants have been reliant on reeds. Such projects are practiced as part of public works projects. In contrast to these projects, reeds have also been harvested and utilized as furniture by residents for a long time as seen in Lake Biwa and the retarding basin in Watarase. That is, the industrial use of reeds for water purification and furniture is well established. However, large quantities of lowcost furniture made of reeds are being imported, which has damaged the domestic reed furniture industry. Public works projects are finding it difficult to cost-effectively maintain reed wetland systems. As a result, the recycle system with reeds is not working well recently.

Therefore, in place of reed, I have noticed forage rice being used since it is still beneficial after its harvest to the farmers. Rice, wheat and corn plants are frequently used in agriculture for the utilization of nutrients in wastewater. In Japan, this is widely practiced because rice is one of the

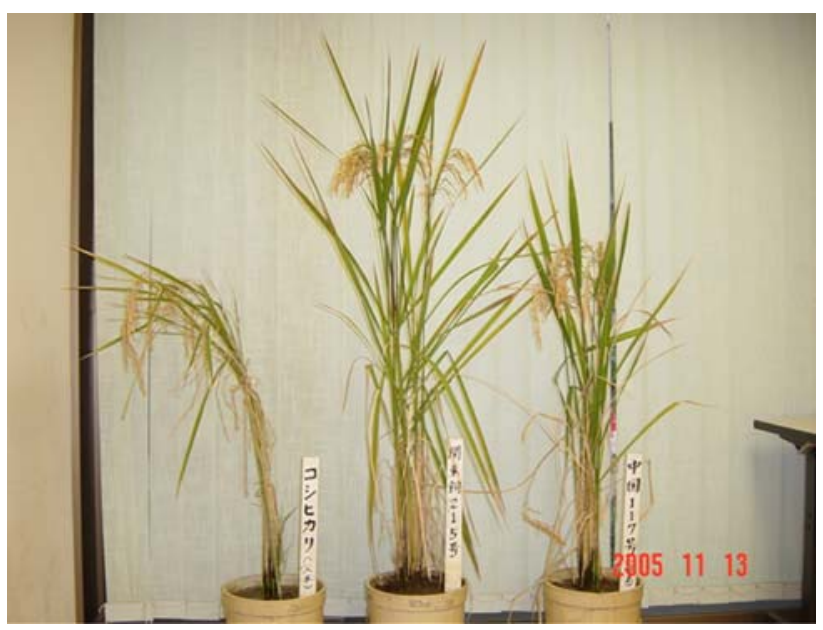

Fig. 1 Comparison of common rice for food (left Koshihikari), Leaf star (middle forage rice), and Chinese rice (right varieties of rice for Leaf star). This picture was provided by Prof. Ookawa 
most important agricultural products. Rice is an important cereal crop in more than 100 countries in the world. Rice is the only crop grown in flooded soil, which is advantageous when treating nutrient-polluted water with a surface-flow system. However, most rice varieties will collapse before harvest, making them difficult to harvest by machine, when a nitrogen loading is higher than the common amount of applied fertilizer (ca. $120-150 \mathrm{~kg} \mathrm{~N} \mathrm{ha}^{-1}$ ). To be able to resist such a high nitrogen loading, new rice varieties have been developed. For example, 'Leaf-star' (Fig. 1), which was developed by Prof. Ookawa and Japan's National Institute of Crop Science (NICS) in 2005, is a new forage rice variety for whole-crop silage (WCS). This kind of forage rice showed dry matter productivity $2-3$ times larger than the general variety in yield trials and tolerance against high nitrogen loading and disease.
Although, for now, "rice paddy" is just a field where rice is produced, it should be changed into a more beneficial place where re-generable energy and biomaterial are newly created while the current function (rice production) is kept. Such technological innovation has great potential on a global scale (especially in Asian countries) since it could achieve low energy consumption. At least in agrarian communities this practice can contribute to a "low carbon society", "natural symbiotic society" and "recycle society" I dream of a time when such symbiotic processes, as learned in paddy field rice growing, can inoculate other areas of human enterprises and lead to many innovations to provide a low carbon society over all. 